\title{
INTERPLANETARY STREAM INTERFACES
}

\section{LEONARD F. BURLAGA}

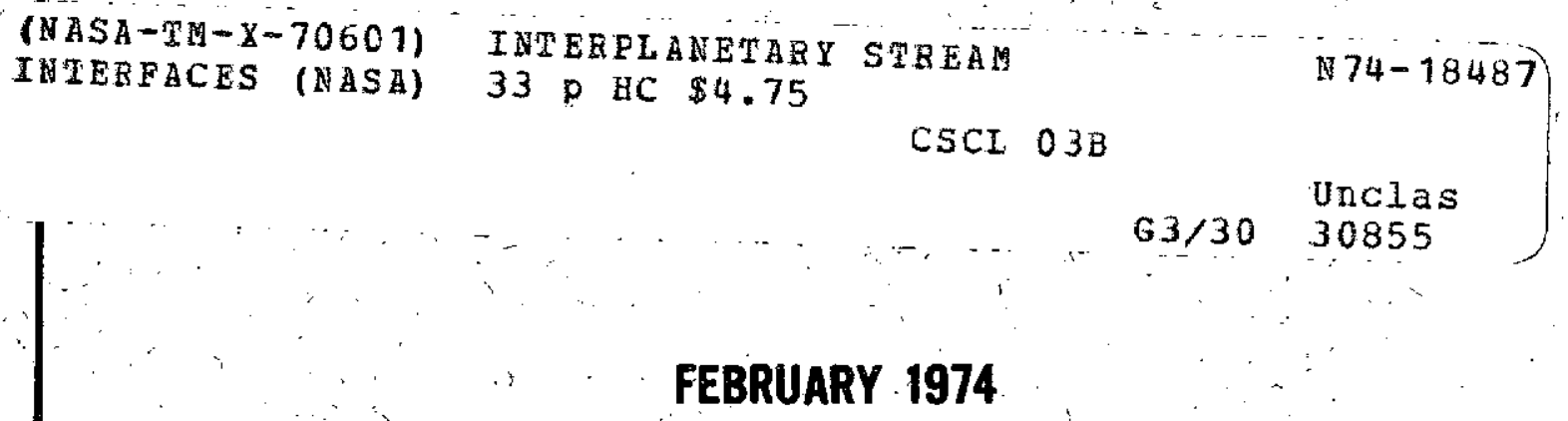

GSFC - GODDARD SPACE FLIGHT CENTER GREENBELT, MARYLANO 


\section{INLERPLANETARY STREAM INTERFACES}

L. F. Burlaga

Laboratory for Extraterrestrial Physics

NASA/Goddard Space Flight Center

Greenbelt, Maryland 20771 


\section{Abstract}

At $1 \mathrm{AU}$ there is a distinct boundary (the stream interface) at the leading edge of a stream in the solar wind, characterized by an abrupt drop in density, a similar increase in temperature and a small increase in speed. In some cases this is a tangential discontinuity; in others it is probably evolving into a tangential discontinuity. It is suggested that stream interfaces form in the interplanetary medium as a consequence of the non-linear evolution of streams generated by an increase in temperature in the solar envelope. This evolution eventually leads to the formation of a reverse shock behind the interface and a forward shock ahead of it. Two instances in which both a stream interface and a reverse shock had developed at I AU are presented. Examples of flare-generated shocks which passed through a stream and were observed near a stream interface are also presented. It is shown that stream interfaces are definitely not the same structures that others have identified as piston boundaries. It is noted that slow shocks, like stream interfaces, always occur ahead of streams and may develop in the interplanetary medium. The importance of small-scale interplanetary dynamical processes, especially the development of discontinuities, is emphasized.

\section{ii}




\section{Introduction}

Since the earliest observations of interplanetary streams (Neugebauer and Snyder, 1966), it has been known that the density and temperature are enhanced at the leading edge of a stream. At 1 AU, the density peak is observed ahead of the temperature peak (Burlaga et al., 1971), because streams are produced by heating in the solar envelope and evolve nonlinearly as they move to the earth (Burlaga et al., 1971; Siscoe and Finley, 1972; Hundhausen, 1972; Goldstein, 1973). This paper shows that at I $\mathrm{AU}$ there is a thin boundary between the density and proton temperature peaks, characterized by an abrupt decrease in a ensity, a similar increase in temperature, and a small increase in bulk speed. This boundary is a striking, clearly recognizable, and apparently rather general feature of streams which is distinct from most other discontinujties in the solar wind. It has an origin which can be understood in terms of stream dynamics, and it may be the site of some interesting instabilities and transport processes, because of the exceptionally large gradients which can develop at the boundary. It also has a special relation to interplanetary shocks. For these reasons, it is appropriate to give the boundary a name. Let us call it the stream interface.

Observations of stream interfaces are presented and some particularly interesting features are noted in the following sections. Some theoretical aspects of stream interfaces and their relations to observations are then discussed. Finally, the observed relations between stream interfaces and shocks are examined. 


\section{Observations of Stream Interfaces}

Existence and Nature. An example of a stream interface is shown in Figure 1. The data are from the GSFC instruments on Explorer 43, which are described briefly in Burlaga and Ogilvie (1973). The interface occurred at 1155 UT on March 23, when the bulk speed V began to increase in front of a stream. At the interface, the density dropped abruptly (in $<3 \mathrm{~min}$ ) from $(11.6 \pm 0.3) \mathrm{cm}^{-3}$ to $(5.6 \pm 0.2) \mathrm{cm}^{-3}$ and the temperature increased similarly from $(4.0 \pm 3) \times 10^{4}{ }^{\circ} \mathrm{K}$ to $(9.0 \pm$ 1.0) $\mathrm{x} 10^{4} \mathrm{~K}$. The discontinuity separates a region of very dense material (Figure 1) from an anomalously hot region (see Figuxe $I$ and Burlaga and Ogilvie, 1973). The magnetic field intensity, B, increased across the interface; but as will be shown, this is not a general characteristic of stream interfaces. The magnetic field direction also changed across the interface, but the change is small. The pressure, $\mathrm{P}=\mathrm{B}^{2} /(8 \pi)+\mathrm{n}_{\mathrm{p}} \mathrm{kT} \mathrm{T}_{\mathrm{p}}+\mathrm{n}_{\alpha} \mathrm{k} \mathrm{T}_{\alpha}+\mathrm{n}_{\mathrm{e}} \mathrm{kT} \mathrm{T}_{\mathrm{e}}$, where $\mathrm{T}_{\mathrm{e}}=1.5 \times 10^{5} \circ \mathrm{K}$, is shown at the top of Figure 1. Since $P$ is continuous across the interface and since $n, T$, and $B$ change appreciably, the stream interface is most probably a tangential discontinuity. The slopes of $P(t)$ and $V(t)$ are continuous across the interface.

Another stream interface is shown in Figure 2, also based on Explorer 43 data. This occurs near 0500 UT on March 31, at a time when the speed began to increase ahead of a stream. The jumps in density and temperature are large (from $\approx(10.5 \pm .3) \mathrm{cm}^{-3}$ to $\approx(4.5 \pm .2) \mathrm{cm}^{-3}$ and from $(1.25 \pm .10) \times 10^{5} \mathrm{~K}$ to $(2.8 \pm 0.1) \times 10^{5} \circ \mathrm{K}$, respectively), but the boundary is thicker than in the previous case, the transition 
occurring in $\approx 30 \mathrm{~min}$. instead of $<3 \mathrm{~min}$. Another difference is that the pressure diminishes continuously through this interface, so it cannot be described as a tangential discontinuity. In this case, the slopes of $P(t)$ and $V(t)$ do change across the interface. The magnetic field intensity does not change through the interface, but the direction fluctuates considerably。

In order to determine whether or not interfaces are a general feature of the solar wind at I AU, plasma data from Ogilvie's instrument on Explorer 34 for the period June 3 to December 16, 1967 were examined in search of stream interfaces. Despite interrupted coverage due to immersion of the spacecraft in the magnetosphere near perigee and despite a great variety of stream patterns (see Burlaga and Ogilvie, 1970 a, Figure 2, for a macroscale plot of $V$ and 1 ), at le ast Il stream interfaces were present, i.e, an average of at least approximately two per solar rotation.

Five of the Explorer 34 stream interfaces are shown in Figure 3. The errors in density and temperature are appreciably larger in the Explorer 34 data than in the Explorer 43 data, and the resulting errors in $P$ are so large that one cannot identify changes as accurately as those in Figure 2. Consequently, only the basic parameters, $V, n, T$, and $B$ are shown in Figure 3. In every case one sees the characteristic signature of a stream interface--a large, abrupt drop in density accompanied by a similar increase in temperature, separating a dense region from a hot one, and occuring at the leading edge of a stream. The change usually occurs at the foot of the stream, but this is not always so as demonstrated by the August 17, 1967, event in Figure 3. 
The profiles of $B(t)$ in Figure 3 support our earlier conclusion that $\mathrm{B}$ does not change in a characteristic way across an interface and that one cannot identify a stream interface from magnetic field data alone. Figure 3 shows a small change in $|\mathrm{B}|$ in some events, but no change in others. Inspection of higher time resolution data revealed. that the direction of $B$ can change discontinuously, smoothly, randomly, or not at all across an interface. In most cases, it is not possible to calculate a "normal" ( $\dot{\mathrm{B}}_{1} \mathrm{x} \underset{\mathrm{B}}{\mathrm{B}}$ ) for an interface using only a stream interface because of fluctuations in $B$, but it is evident that ${\underset{Z}{I}}_{I}$ and $\underset{\sim}{B}$ tend to lie more nearly along the spiral direction than perpendicular to it or radially. This is consistent with the stream interfaces being surfaces which extend along the spiral direction.

One might expect that a stream interface would be recorded as an $\mathrm{SI}^{-}$by geomagnetic observatories, since the decrease in density implies a drop in momentum flux (see the review by Burlaga, 1972). However, an examination of magnetograms from 12 stations for each of the events in Figure 3 revealed that in no case did a distinct S. $^{-}$occur at all stations simultaneously. 


\section{Theory}

The observations just presented raise several theoretical questions. Do the large gradients in $\mathrm{n}$, $\mathrm{T}$, and $\mathrm{B}$ cause instabilities? Is the bulk speed jump across the interface due to a shear; and if so, can this generate the Kelvin-Helmholtz instability? How is heat transported across the interface? These interesting questions will be left unanswered, but some basic questions concerning the speed of the interface, the change in $P$ and $V$ across the interface, and its origin will now be considered.

Motion. Let us approximate the interface by a discontinuity moving with respect to the sun with speed $U$, and let the pressure be $P_{I}$ ahead (early time) and $P_{2}$ behind (later time), respectively. Let $V$ be the solar wind speed with respect to the sun. Conservation of mass and conservation of momentum flux give $e_{I}\left(V_{I}-U\right)=e_{2}\left(V_{2}-U\right)$ and $e_{I}\left(V_{I}-U\right) x$ $\left(V_{2}-V_{1}\right)=P_{1}-P_{2}\left(e . g_{0}\right.$, see Burlaga, 1971 .q. 5.1 and Eq. 5.3) from which one obtains for the speed of the interface relative to the speed of the plasma ahead of it

$$
U-V_{1}=\sqrt{\frac{\rho_{2}}{P_{1}} \frac{\left(P_{1}-P_{2}\right)}{\left(P_{1}-e_{2}\right)}}
$$

If $P_{1}=P_{2}$, as for the interface shown in Figure 1 , then $U=V_{1}$, and the interface is simply convected with the plasma, consistent with the earlier statement that this interface is a tangential discontinuity.

If $P_{1} \neq P_{2}$, the interface will move with respect to the plasma ahead of it. This should be the case for the interface shown in Figure 2. Putting the observed values of $P_{1}, P_{2}, e_{1}$, and $e_{2}$ into $(1)$, 
gives $U-V_{I}=35 \pm 6 \mathrm{~km} / \mathrm{sec}$. The observed speed change across the interface is $V_{2}-V_{I}=56 \pm 7 \mathrm{~km} / \mathrm{sec}$. Thus, $U-V_{I}$ is approximately equal to the mean speed $\left(V_{2}-V_{1}\right) / 2$. One can regard the motion of the interface as a consequence of the overtaking of the slower part of the stream by the faster part. If the steepening continues without resistance, the interface will evolve from a boundary with thickness $D \approx 5 x$ $10^{5} \mathrm{~km}$ at $1 \mathrm{AU}$ to a discontinuity in a time on the order of $\mathrm{D} /\left(\mathrm{V}_{2}-\mathrm{V}_{1}\right)$, which is a few hours and corresponds to a distance of a few hundredths of an AU beyond. 1 AU. Thus, one expects the interface in Figure 2 to evolve into one like that in Figure 1 . This is one way in which tangential discontinuities can form in the solar wind.

Jump condition. Figure 2 shows that the slopes of $P(t)$ and $V$ (t) can change across stream interface. A relation between the change in $\partial \mathrm{P} / \partial \mathrm{t}$ and $\partial \mathrm{V} / \partial \mathrm{t}$ is sbtained as follows.

Since" the interface passes a fixed observer in a matter of minutes, which is much smaller than the characteristic time of the stream evolution, the pressure of a small volume element containing the interface does not change significantly as it passes the observer, and

$$
\frac{d P}{d t}=\frac{\partial P}{\partial t}+(V \cdot \nabla) P \approx 0
$$

Thus, at a spacecraft,

$$
\frac{\partial P}{\partial t}=-\left(v_{x} \frac{\partial P}{\partial x}+v_{y} \frac{\partial P}{\partial y}+v_{z} \frac{\partial P}{\partial z}\right)
$$

where $(x, y, z)$ are solar ecliptic coordinates, $\hat{x}$ pointing toward the sun and $\hat{z}$ toward the north ecliptic pole. In general, near I AU, $\frac{\partial P}{\partial y} \approx \frac{\partial P}{\partial x}$, but $V_{y} \ll V_{x}$ so $V_{y} \frac{\partial P}{\partial y}$ is small compared to $V_{x} \frac{\partial P}{\partial x}$. Similarly, 
$V_{z} \frac{\partial P}{\partial z}$ is probably negligible. Thus,

$$
\frac{\partial P}{\partial t} \approx-V_{x} \frac{\partial P}{\partial x} \approx-V \frac{\partial P}{\partial x}
$$

where $V$ is the measured bulk speed. This simply says that the measured time variations of $P$ are actually the result of the convection of a non-uniform but stationary pressure profile. A similar relation holds for $\frac{\partial V}{\partial t}$. The equation of motion gives

$$
V_{x} \frac{\partial V_{x}}{\partial x}+V_{y} \frac{\partial V_{y}}{\partial y}+V_{z} \frac{\partial V_{z}}{\partial z}=-\frac{1}{\rho} \frac{\partial P}{\partial x}
$$

Now $\frac{\partial V_{x}}{\partial y}$ and $\frac{\partial V_{x}}{\partial z}$ can be comparable to $\frac{\partial V_{x}}{\partial x}$, but $V_{z} \ll V_{x}$ and $V_{y}<V_{x}$. Thus,

$$
V \frac{\partial V}{\partial x} \approx-\frac{1}{\rho} \frac{\partial P}{\partial x}
$$

Using (2) and the corresponding relation for $\frac{\partial V}{\partial t}$ we obtain

$$
\frac{\partial V}{\partial t}=-\frac{1}{\rho V} \frac{\partial P}{\partial t}
$$

which gives the desired jump condition

$$
\left.\frac{1}{\mathrm{eV}} \frac{\partial \mathrm{P}}{\partial t}\right|_{1} ^{2}=-\left.\frac{\partial V}{\partial t}\right|_{1} ^{2}
$$

In general, $\frac{\partial V}{\partial t}$ and $\frac{\partial P}{\partial t}$ can change across the interface even if $P$ and $V$ do not. If there is no change in $\frac{\partial V}{\partial t}$ across the interface, then $\frac{I}{\mathrm{eV}} \frac{\partial \mathrm{P}}{\partial t}$ must be continuous across the boundary. This is the case for the interface in Figure 1 , where $\frac{\partial V}{\partial t}$ and $\frac{\partial P}{\partial t}$ are zero on both sides of the boundary. In the case of the interface in Figure $2, \frac{\partial V}{\partial t}<0$ ahead of the interface and $\frac{\partial V}{\partial t}>0$ behind, so that $\left.\frac{\partial V}{\partial t}\right|_{1} ^{2}<0 ; \quad \frac{\partial P}{\partial t}<0$ behind while $\frac{\partial P}{\partial t}>0$ ahead, giving $\left.\frac{1}{e V} \frac{\partial P}{\partial t}\right|_{1} ^{2}<0$. This agrees with (3). Quantitatively, using the slopes drawn in Figure 2, one finds that the RHS of (3) is $30 \%$ smaller than the LHS, but the differences is 
wishin the uncertainties.

Origin. The explanation of the origin of stream interfaces is implicit in the model results of Hundhausen (1973). This is the same model which Burlaga et al. (1971) used to explain the separation between the temperature peak and density peak in front of a stream, extended to examine the evolution of a stream at later times and larger distances from the sun. It is based on the equations which Hundhausen and Gentry (1969) integrated to follow the motion of a shock, but uses a different condition for the variation of the temperature at the inner boundary. Hundhausen's solution for the density and speed, pressure, and temperature profiles (Hundhausen, 1973 Figures 2, 3, 4, and 5) show that three discontinuities form somewhere between I AU and $1.5 \mathrm{AU}$. One, characterized by an increase in $\mathrm{n}, \mathrm{T}$, and $\mathrm{V}$, develops into a forward shock. This is followed by a second discontinuity across which the density decreases and temperature increases, and which occurs at the leading edge of the stream, behind the velocity jump associated with the shock; this clearly has the signature of a stream interface. The third discontinuity is characterized by a decrease in $\mathrm{n}$ and $\mathrm{T}$ and an increase in $\mathrm{V}$; this evolves into a reverse shock. Unfortunately, Hundhausen's published results do not show the detailed development of these discontinuities near 1 . AU. Nevertheless, the results show that a discontinuity with characteristics of a stream interface is a natural consequence of the dynamical evolution of a stream which originates from an increase in temperature near the sun $(0.133 \mathrm{AU}=28 \mathrm{R})$ and moves into a quiet solar wind. From the resul.ts of Hundhausen (1973), one expects that beyond $\approx 1.5$ AU, stream interfaces will always be associated with shock pairs. The 
three discontinuities need not form simultaneously, however. For example, Formisano and Chao (1972) show how the reverse shock can form before the forward shock. Thus, the sequence of development is likely to be stream interfacereverse shock-fast shock (see Figure 4). The actual positions at which the discontinuities form will change from one event to the next.

The data which we examined showed that stream interfaces are usually not observed in association with shocks at 1 AU. Our interpretation implies that somewhere beyond I AU but $\leqslant 1.5$ AU reverse shocks will always be observed with stream interfaces. One might expect to see such a result occasionally even at 1 AU. Such observations and other shock associations are discussed in the next section. 
Relations between Stream Interfaces and Shocks

Reverse Shocks and Stream Interfaces. Burlaga (1970) identified a reverse fast MFD shock in Explorer 34 data and noted that this occurred at a fast stream. These observations are shown in Figure 5. Formisano and Chao (1972) suggested that this formed by the steepening of a stream, essentially by the same process described in more detail by Hundhausen (1973). They suggested that a forward shock was also developing, consistant with the general idea that shock pairs should form at streams. The orientation of this shock surface (along the spiral direction) is consistent with this mechanism. Here we make the additional observation that ahead of the shock (at $\approx 0700$ UT on September 28, 1967) there is another boundary, having the signature of a stream interface. The boundary is actually quite distinct, the density and temperature changing appreciably in less than three minutes.

Chao et al. (1973) have recently published evidence of another reverse fast shock which they suggested is associated with a discontinuity that is developing into a forward fast shock (see Figure 6). The two "shocks" are at the two ends of the pressure pulse ahead of a stream, and the reverse shock is oriented in the spiral direction. Thus, the two discontinuities appear to be formed by the interaction of the stream with the material ahead of it, as suggested by chao et al. Here we make the additional observation that between the reverse shock and the developing forward shock is a stream interface.

The two examples just discussed are clearly consistent with our view that stream interfaces originate in the solar wind as the result 
of the steepening of a stream.

Stream Interfaces and Flare Associated Shocks. Since fast, forward shocks can be generated by flares as well as by streams, and since flaregenerated shocks can move through streams.(Ogilvie and Burlaga, 1974), one might expect to find "coincidental":" associations between stream interfaces and fast forward shocks, thereby complicating the interpretation of shock ensembles. Here we show two such coincidental associations.

Figure 7 shows a stream interface at 0345 UT on November 29, 1967 which is followed by a fast, forward, MFD shock. The shock was not produced by the stream, since it did not occur ahead of the stream and. the pressure pulse. Evidently, the shock simply happened to be propagating through the stream.

Another example of a forward fast shock associated with a stream interface is shown in Figure 8. In this case, the shock precedes the interface, so it is possible that they are causally related. However, Ogilvie and Burlaga (1974) concluded from a detailed analysis of this event that the shock was probably generated by a flare and simply passed through the stream. Thus, the association of the fast shock and stream interface is coincidental in this case, too.

Slow Shocks. Here we digress briefly to discuss another feature in Figure 8. Behind the stream interface in Figure 8 is a discontinuity which has the signature of a slow, forward MHD shock, i.e., B decreases while $\mathrm{n}, \mathrm{T}, \mathrm{U}$, and $\mathrm{P}$ increase (e.g., see Burlaga, 1971 for a general discussion of such shocks). However, a detailed analysis of this event, using several sets of parameters consistent with the measurements and 
their uncertainties for the states before and aftex the discontinuity, shows in every case that the flow speed does not exceed the slow mode speed of the discontinuity. Thus, the event is not a slow shock. Since there is no other MFD discontinuity with such a signature and with $P_{1} \neq$ $\mathrm{P}_{2}$, we must conclude that either MFD is not applicable or the structure is not a stationary discontinuity. There is no strong reason to deny the applicability of MFD on this scale, since it satisfactorily describes many of the discontinuities that have been observed (Burlaga, 1971). On the other hand, the structure is rather broad and occurs in a very dynamic situation, so it is reasonable to consider that it is just evolving into a slow shock.

The "slow shock" just described occurs at the leading edge of a stream. In fact, all of the slow shocks which have been reported in the literature occux at the leading edge of a stream. This point is demonstrated in Figure 9 which shows plots of the bulk speed and the location with respect to the streams of the slow shocks for each of the five known slow shocks. The location of slow shocks suggests that they are formed in the interplanetary medium by stream interactions. The theory of this process has not been developed. The slow shocks shown in Figure 9 are reported in Chao and Olbert (1970), Burlaga and Chao (1974), Mikalov et al. (1973). 
Relations between Piston Boundaries and Interplanetary Stream

Interfaces. Several authors have suggested that some or all flare associated shocks are driven by a jet of gas (the "piston") and that the front of this jet is a sharp boundary. The model was formulated quantitatively by Parker (1963). Observations of this boundary have been elusive, however. Hirshberg et al. (1970) and Unti et al. (1973) tentatively identified such a surface using plasma and magnetic field data. Some papers on cosmic ray propagation (e.g. Quenby, 1971 ; Barnden, 1973) attempt to identify this surface using only magnetic field and cosmic ray data. Here we consider whether or not stream interfaces are related to the piston boundaries.

Hirshberg et al. (1970) identified a piston boundary as an abrupt increase in density, an increase in $\mathrm{n}_{\alpha} / \mathrm{n}_{\mathrm{p}}$, and a decrease in $|\mathrm{B}|$. Unti et al. (1973) chose a discontinuity with a similar signature; an increase in $n, n_{\alpha} / n_{p}$ and a small drop in $|\underset{\sim}{B}|$, and also noted an increase in T. Barnden (1973) used only magnetic field data, but found that $B$ dropped across each of the several piston boundaries which he identified. Quenby (1971), using only magnetic field observations, identified a piston boundary for one event as an abrupt decrease in B. All of the above results are consistent in that they find a decrease in $B$ across the piston boundary. This alone, however, indicates that these boundaries are probably not stream interfaces, because B does not usually drop across an interface. The results of Hirshberg et al. (1970) and Unti et al. (1973) are consistent in that both see a density increase, but this also excludes a stream interface, since the density 
always drops across an interface. Finally, while Hirshberg et al. and Unti et al. find an increase in $n_{\alpha} / n_{p}$, the two interfaces for which we have $\alpha$-particle data show no such increase. In summary, the piston boundaries which have been identified in the papers listed above are not stream interfaces. This is consistent with the view that stream interfaces are the result of interplanetary dynamical processes rather than flares. 


\section{Summary}

It was shown that in general there is a distinctive boundary at the leading edge of a stream at $1 \mathrm{AU}$, called the stream interface, Which separates a region of high density from one of high proton temperature. It is characterized by an abrupt drop in density, a similar rise in temperature, and a small increase in bulk speed. In some cases the interface is stationary and has the nature of a tangential discontinuity, In other cases the pressure changes across the interface, which presumably moves with respect to the plasma ahead of it; it is suggested that such an interface evolves into a tangential discontinuity just beyond $1 \mathrm{AU}$. When the interface is in equilibrium, the pressune $P$ is constant across it but the slope of $P(t)$ may change.

Stream interfaces probably originate in the interplanetary medium as a result of the non-linear steepening of streams generated by an increase of the temperature in the solar envelope. Such a process separates the density and temperature pulses, between which the stream interface forms (Burlaga et a.1., 1971), and it ultimately leads to a pressure pulse within which the stream interface appears and which is bounded by a shock pair (Hundhausen, 1973). Near I AU the shocks are usually not yet developed, and only the stream interface is observed. Presumably a reverse shock begins to form (Formisano and Chao, 1972) slightly beyond I AU. Farther from the sun a fast forward shock also develops.

We showed two cases in which a stream interface preceded a reverse fast shock. In one of these cases the stream interface and a 
reverse shock followed a discontinuity which appeared to be developing into a forward shock. These results are consistent with the model described in the preceding paragraph.

One must be careful in analyzing stream interfaces associated with fast shocks, because in some cases the association may be coincidental. In particular, we showed two cases in which a flare generated shock happened to be passing through a stream near a time when a stream interface was observed. There is also the possibility of confusing stream interfaces with "piston boundarjes" (e.g., Hirshberg et al., 1970), but in all of the cases studied in this paper, stream interfaces have characteristics which are distinctly different from those of piston boundaries.

our results indicate that a variety of ensembles of discontinuities can be observed in the interplanetary medium. The basic results of this paper are schematically illustrated in Figure 4, which shows a stream interface, reverse shock, and forward shock developing ahead of a stream (in that order) interacting with a driven flare-generated shock followed by a "piston boundary." Even more complicated configurations are possible in practice, since other discontinuities are normally present in the solar wind (e.g., Burlaga, 1971) and still others can form as shocks interact with all of the discontinuities that may be present (Parker, 1963).

Finally, we stress the importance of dynamical processes on a small scale. Although the microscale tends to be in equilibrium (Burlaga and Ogilvie, $1970 \mathrm{~b}$ ), this is not always the case. One 
cannot always assume that an abrupt change can be identified with one of the MFD discontinuities, since the later are derived for steady state conditions whereas the former might be undergoing dynamical changes. On the other hand, tangential discontinuities, forward and reverse fast shocks, and slow shocks can form in the interplanetary medium as a result of such processes. 


\section{Acknowledgements}

The plasma and magnetic field data used in this study are from the experiments of Drs. K. W. Ogilvie and N. F. Ness, respectively. Helpful comments on a draft of this manuscript were provided by Drs. R. Lepping, J. Scudder, K. Ogilvie, and E. Barouch. Dr. J. Chao contributed to the analysis of slow shocks. 


\section{References}

Barnden, L. R., The Irarge-Scale Magnetic field configuration associated with Forbush decreases, to be published, 1973.

Burlaga, Ir.F., Directional Discontinuities in the Interplanetary Magnetic Field, Solar Physics, 7, 541969.

Burlaga, L. F., A Reverse Hydromagnetic Shock in the Solar Wind, Cosmic Electrodynamics, $1,233,1970$.

Burlaga, L. F., Hydromagnetic Waves and Discontinuities in the Solar Wind, Space Sci. Rev., 12, 600, 1971.

Burlaga, L. F., Discontinuities and Shock Waves in the Interplanetary Medium and Their Interaction with the Magnetosphere, Solar Terrestrial Physics/1970: Part II, 135, ed. Dyer, D. Reidel, DordrechtHolland, 1972 .

Burlaga, L. F. and J. K. Chao, Reverse and Forward Slow Shocks in the Solar Wind, J. Geophys. Res., 76, 7516, 1971.

Burlaga, L. F. and K. W. Ogilvie, Heating of the Solar Wind, Astrophys. J., 159, 659, 1970a.

Burlaga I. F. and K. W. Ogilvie, Magnetic and Thermal Pressures in the Solar Wind, Solar Physics, 15, 61, 1970 b.

Burlaga, I. F. and K. W. Ogilvie, D. H. Fairfield, M. D. Montogmery, and S.J. Bame, Energy Transfer at Colliding Streams in the Solar Wind, Astrophys. J., 164, 137, 1971.

Chao, J. K., Lepping, R. L., and J. Binsack, A Reverse Shock Associated with a Stream-Stream Interaction: The February 29th, 1968, Event, NASA/GSFC X-692-73-246, submitted to J. Geophys. Res., 1973.

Chao, J. K. and S. Olbert, Observation of Slow Shocks in Interplanetary Space, J.Geophys. Res., 75, 6394, 1970.

Formisano, V. and. J.K. Chao, On the Generation of Shock Pairs in the Solar Wind, Cosmic Plasma Physics, p. 103, ed. Karl Schindler, Plenum Press, New York - London, 1972.

Goldstein, B., Nonlinear Corotating Solar Wind Structure, submitted to Astrophys. J., 1973.

Hirshberg, J., A. Alksne, D. S. Colburn, S. J. Bame, and A. J. Hundhausen, Observation of a Solar Flare Induced Interplanetary Shock and Helium Enriched Driver Gas, J. Geophys. Res., 75, I, 1970. 
Hundhausen, A. J., Coronal Expansion and Solar Wind, Springer-Verlag, New York, 1972 .

Hundhausen, A. J., Nonlinear Model of High-Speed Solar Wind Streams, J. Geophys. Res., 78, 1528, 1973.

Hundhausen, A. J., and R. A. Gentry, Numerical Simulation of FlareGenerated Disturbances in the Solar Wind, J.Geophys. Res., 74,2908 , 1969.

Mihlov, J. D., D. S. Colburn, B. F. Smith, C. P. Sonett, and J. H. Wolfe, Pioneer PIasma and Magnetic Field Measurements in

Interplanetary Space during August 2-17, 1972, preprint, 1973.

Neugebauer, M. and C.W. Snyder, Mariner II Observations of the Solar Wind, 1. Average Properties, J. Geophys. Res., 71, 4469, 1966.

Ogilvie, K. W., and I. F. Burlaga, A Discussion of Interplanetary PostShock Flows with Two Examples, J.Geophys. Res., In press, 1974.

Parker, E. N., Interplanetary Dynamical Processes, John Wiley, New York, 1963.

Quenby, J. J., The Mechanism for the Forbush Decreases in Particular for the Event of February 26, 1969, Proc. 12th Intl. Conf。 Cosmic Rays, Hobart, $2,730,1971$.

Siscoe, G. L., and L. T. Finley Solar Wind Structure Determined by Corotating Coronal Inhomogeneities, 2, Arbitrary Perturbations, J. Geophys. Res., 77, 35, 1972 .

Unti, T., M. Neugebauer, and C.S.Wu, Shock System of February 2, 1969, J. Geophys. Res., 78, 7237, 1973. 
Figure Captions

Fig. 1. A stream interface across which $P$ does not change. Note the decrease in $n$, the increase in $T$ and $V$, and the position with respect to the stream.

Fig. 2. A stream interface across which $P, d P / d t$, and dV/dt change.

Fig. 3. Additional examples of stream interfaces. In every case one sees a decrease in $\mathrm{n}$, an increase in $\mathrm{T}$ and $\mathrm{V}$, and the stream interface occurs in front of a stream. There is no systematic change in $\underset{\sim}{\mathrm{B}}$.

Fig. 4. Origin of stream interfaces. It is suggested that stream interfaces originate in the interplanetary medium as a result of the non-linear steepening of streams generated by an increase in temperature in the solar envelope. This same process eventually leads to the development of a reverse shock and a forward shock, probably in that order. A flare-generated shock is also shown propagating through the stream.

Fig. 5. This is a case where the process described in Figure 4 has evolved to the point where two discontinuities have formed ahead of a stream (the stream interface and a reverse fast shock).

Fig. 6. Another case of a reverse shock and a stream interface ahead of a stream at 1 AU. In this case one might also be seeing a forward fast shock in the process of development in a way consistent with the model in Figure 4.

Fig. 7. An example of a flare-generated shock which is propagating through a stream and happens to be near a stream interface.

Fig. 8. Another example of a flare-generated shock which is propagating through a stream. In this case it has probably passed through the stream interface which is shown. Another discontinuity is shown which appears to be developing into a slow shock.

Fig. 9, This shows the position with respect to streams of all the slow shocks reported in the literature. Slow shocks always develop in front of a stream. 

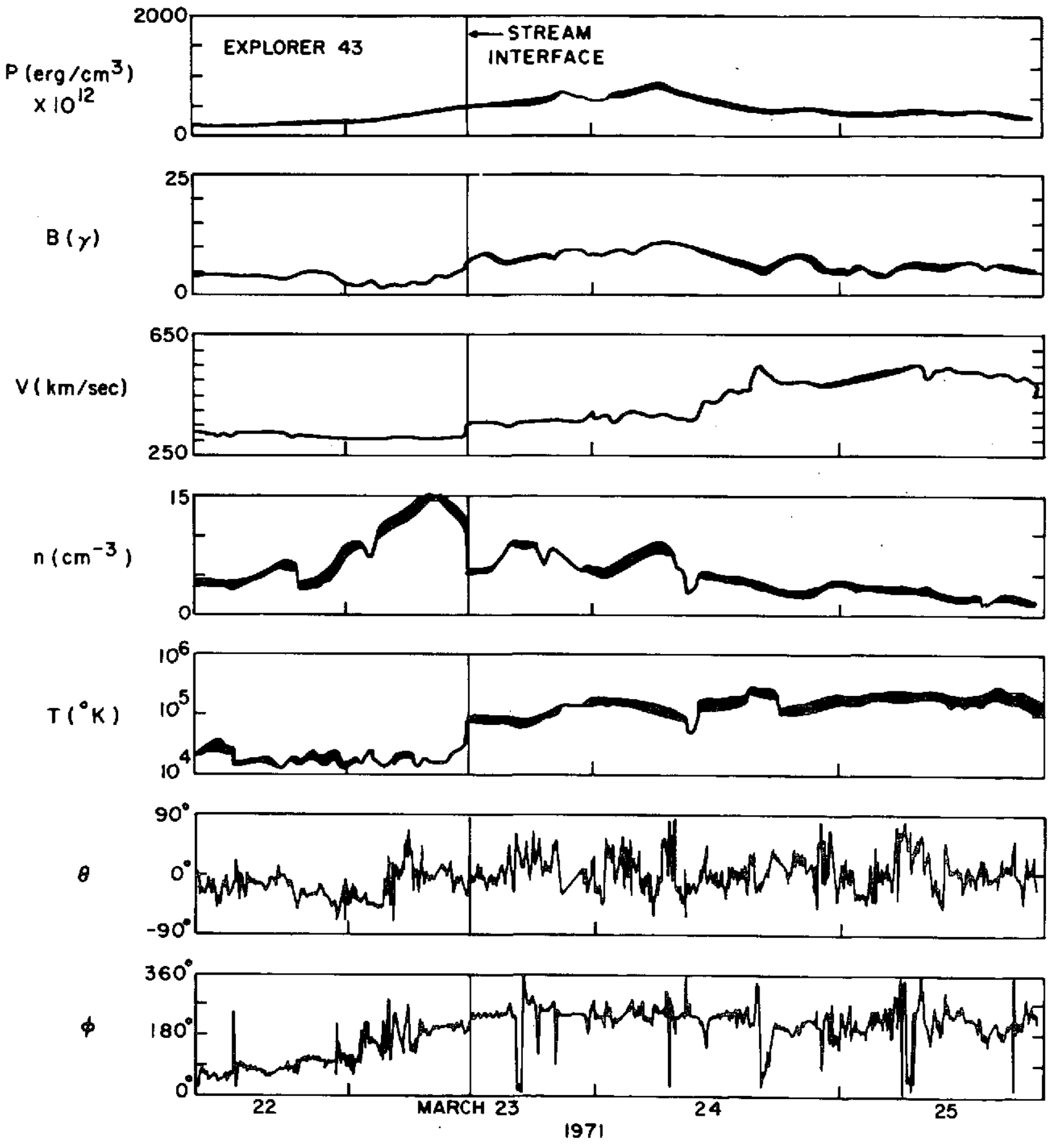

Figure 1 


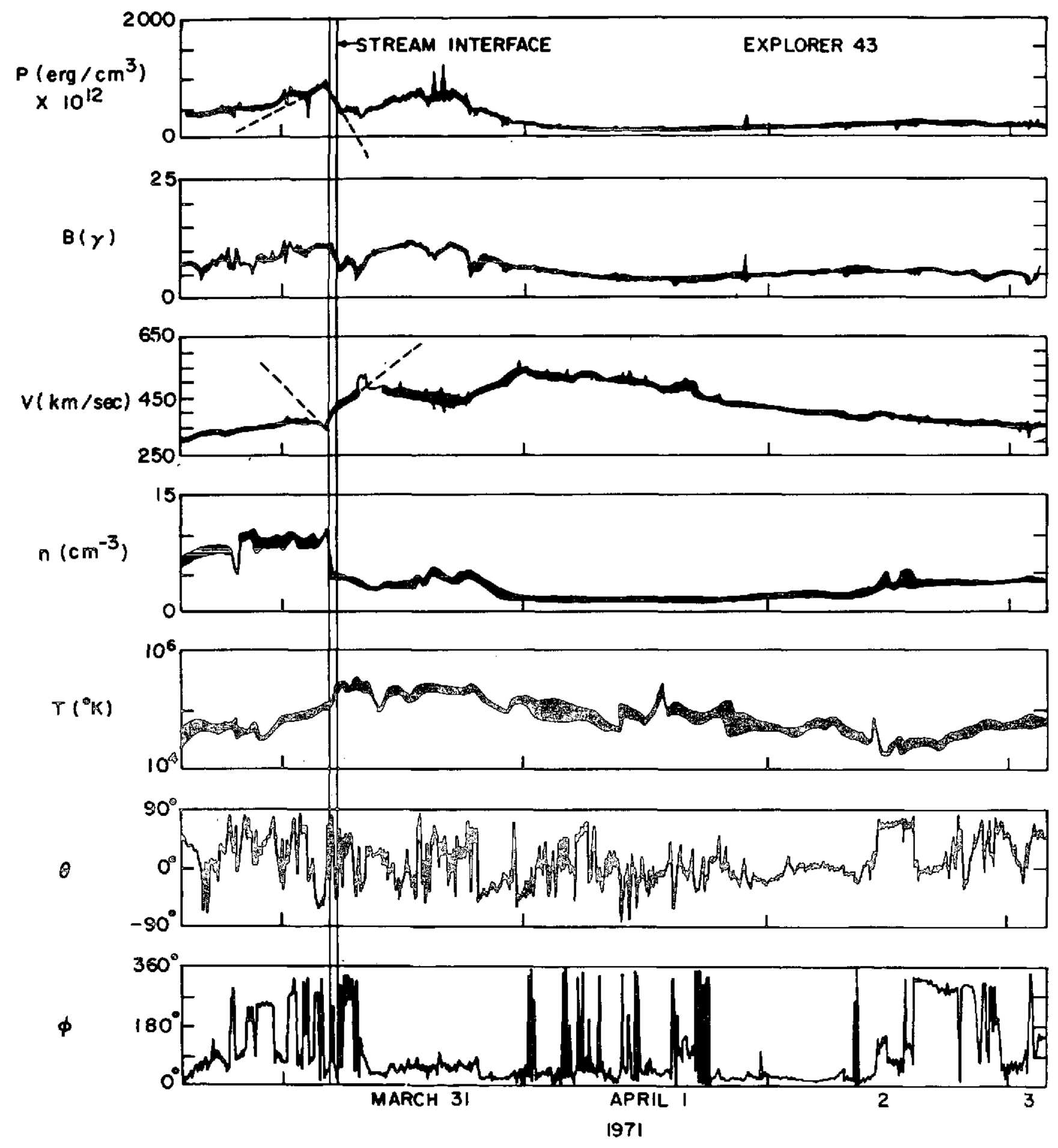

Figure 2 
STREAM INTERFACES - EXPLORER 34
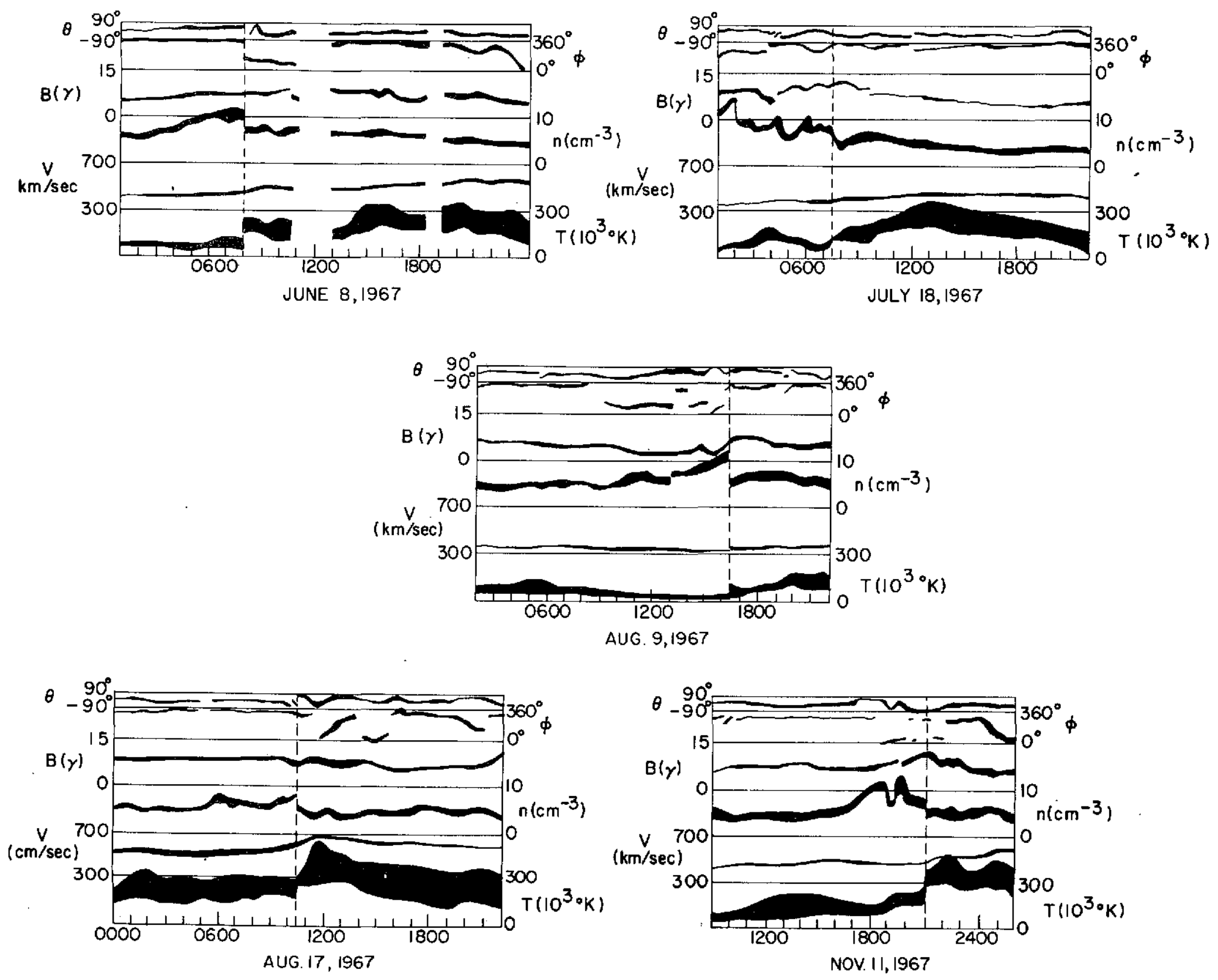


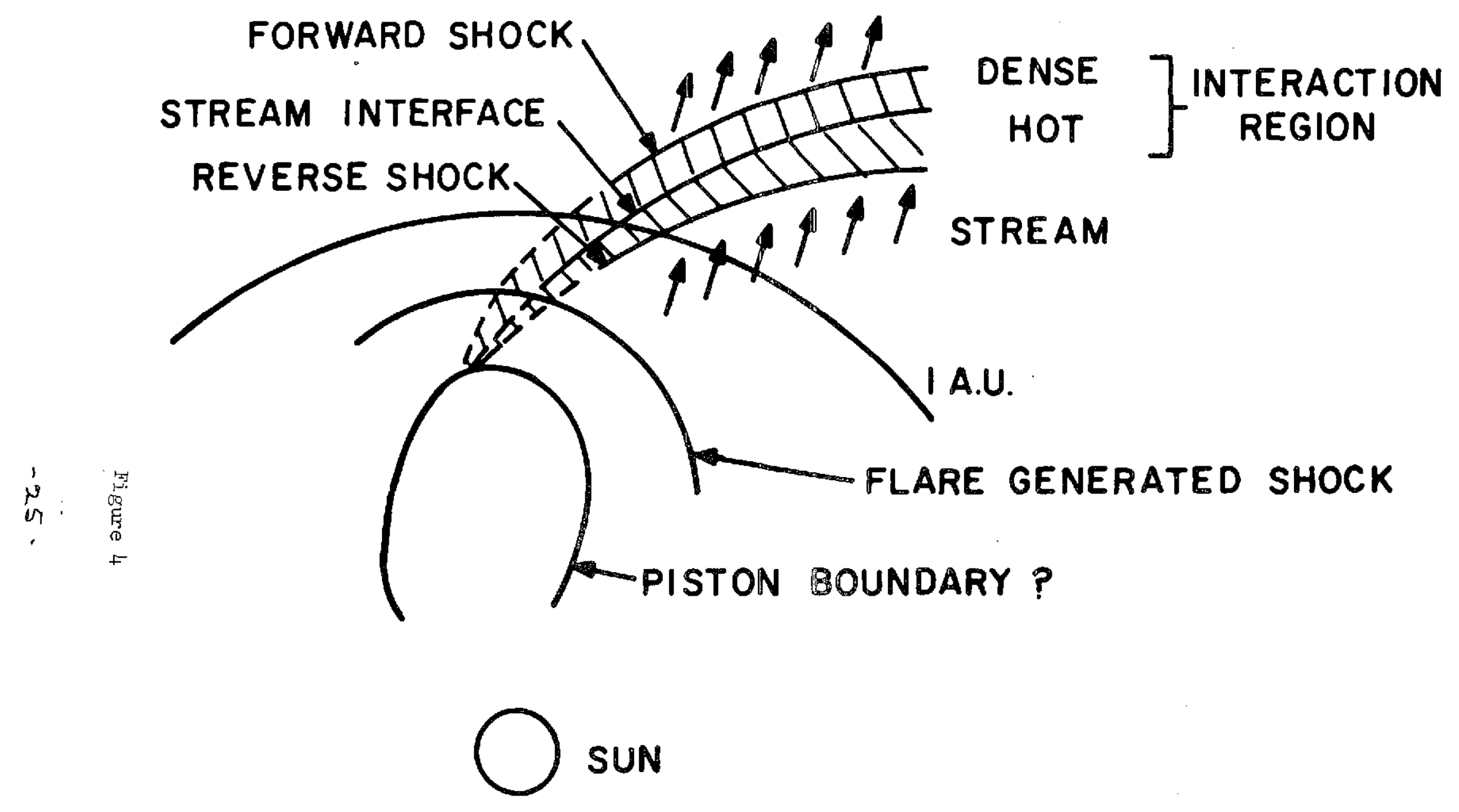




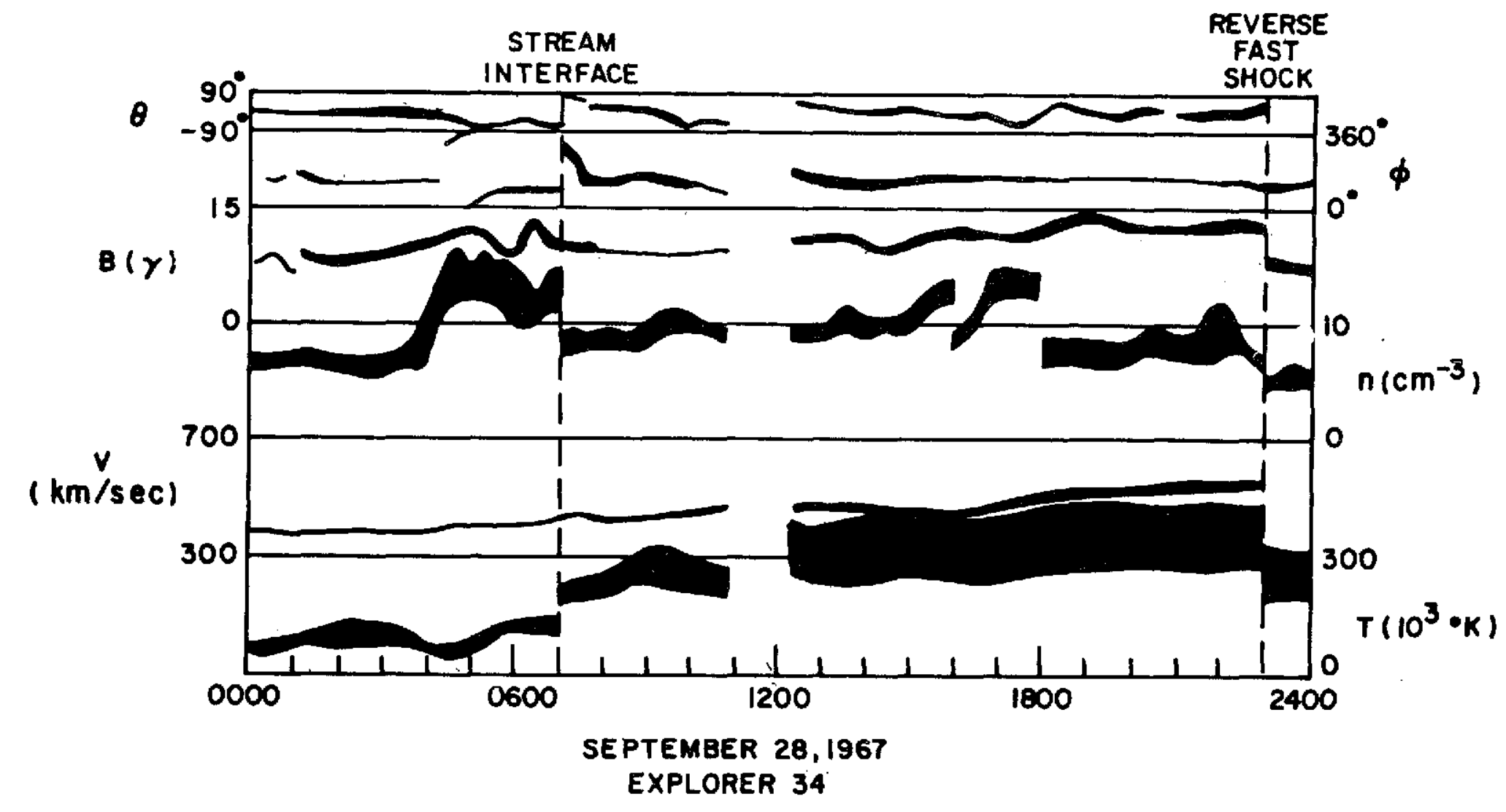

Figure 5 


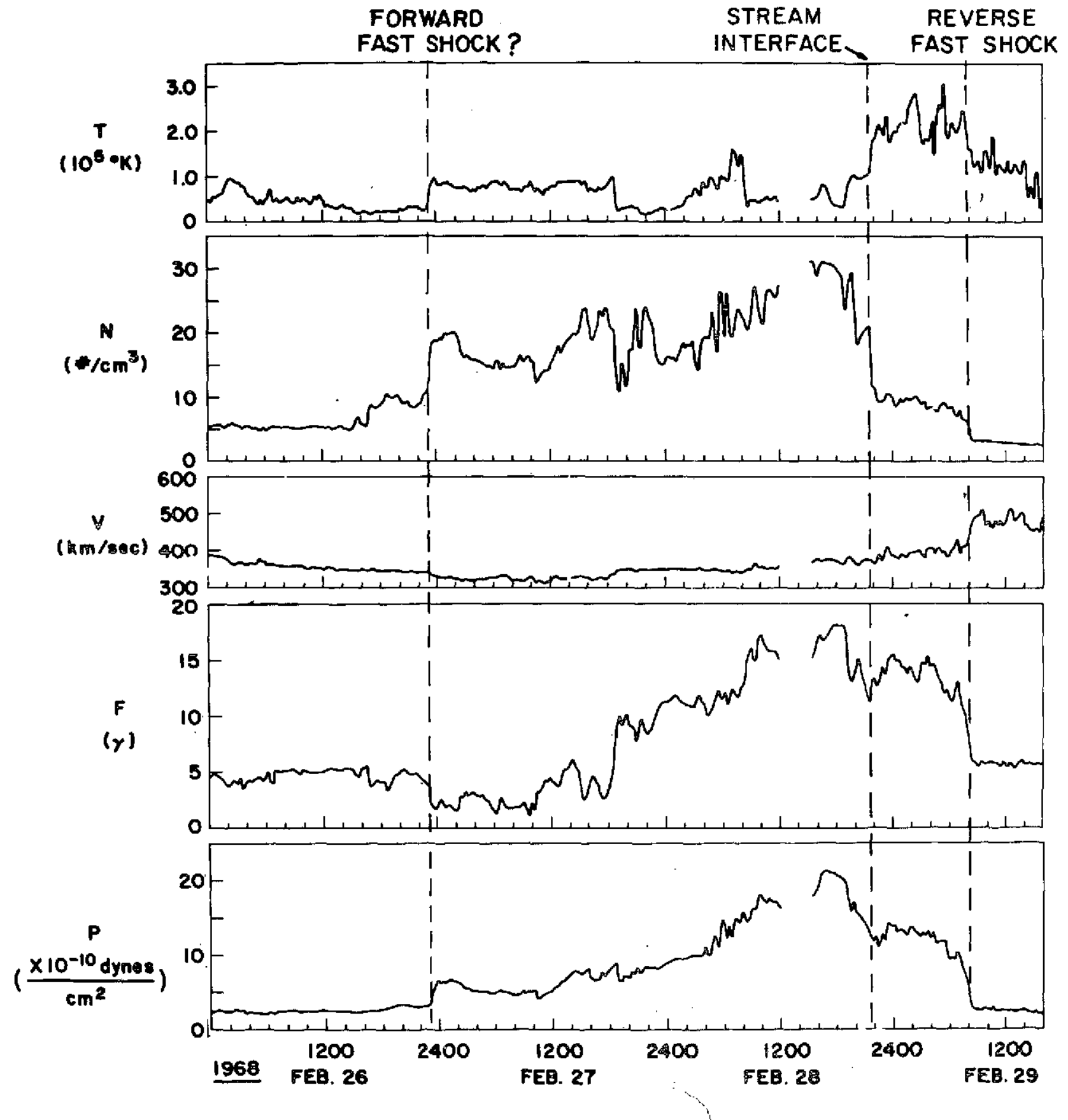

Figure 6 


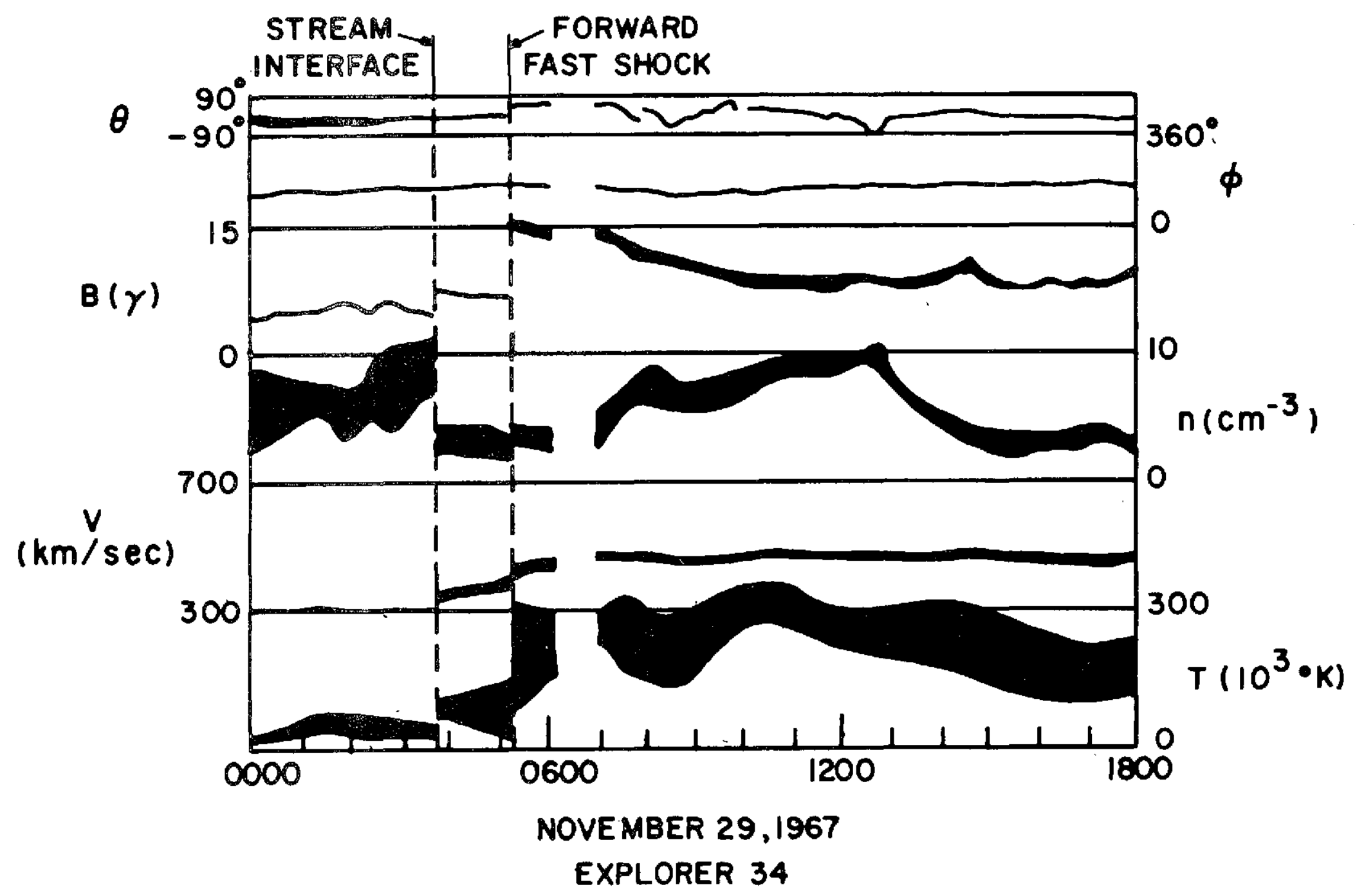

Figure 7 


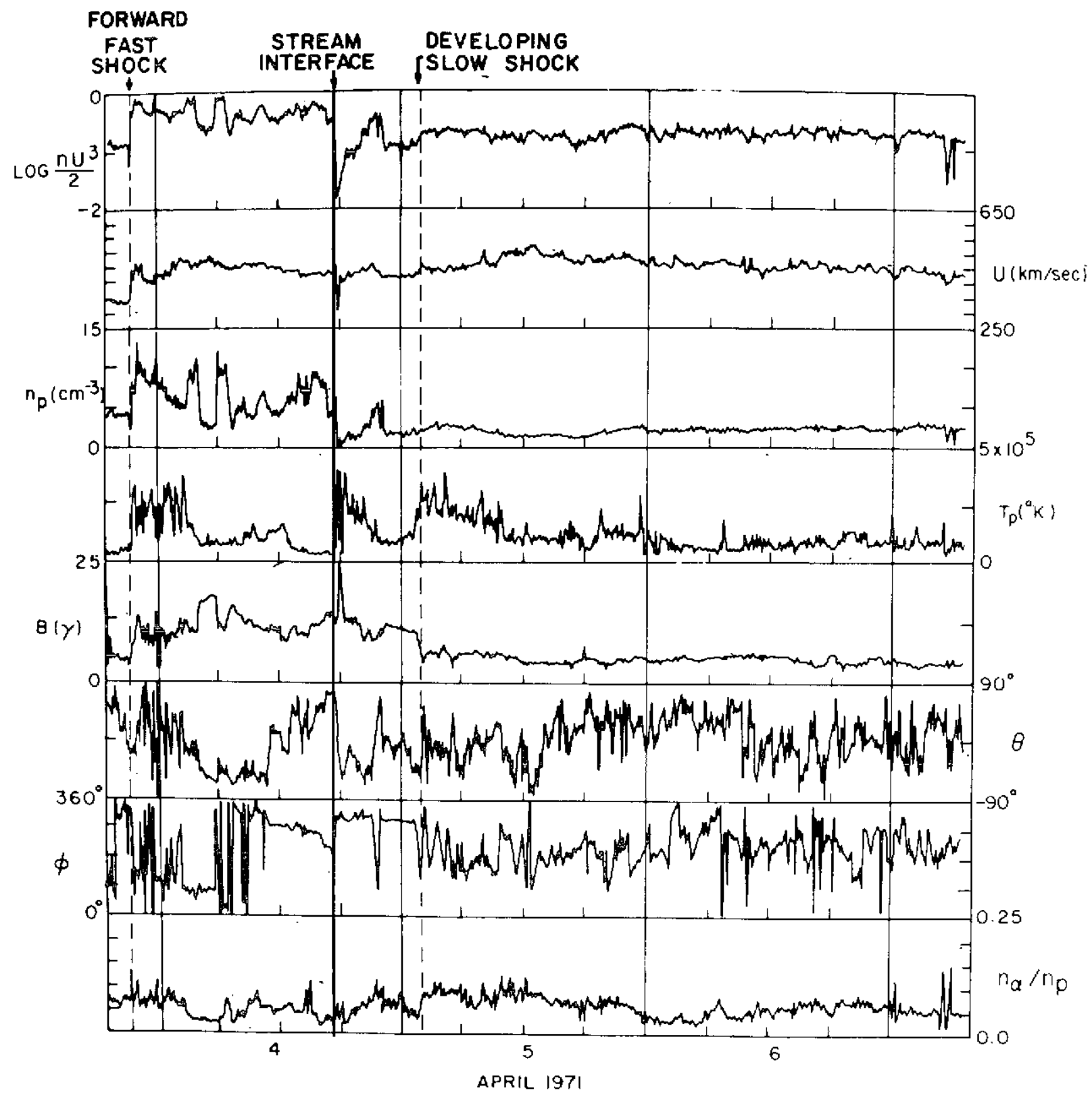

Figure 8 

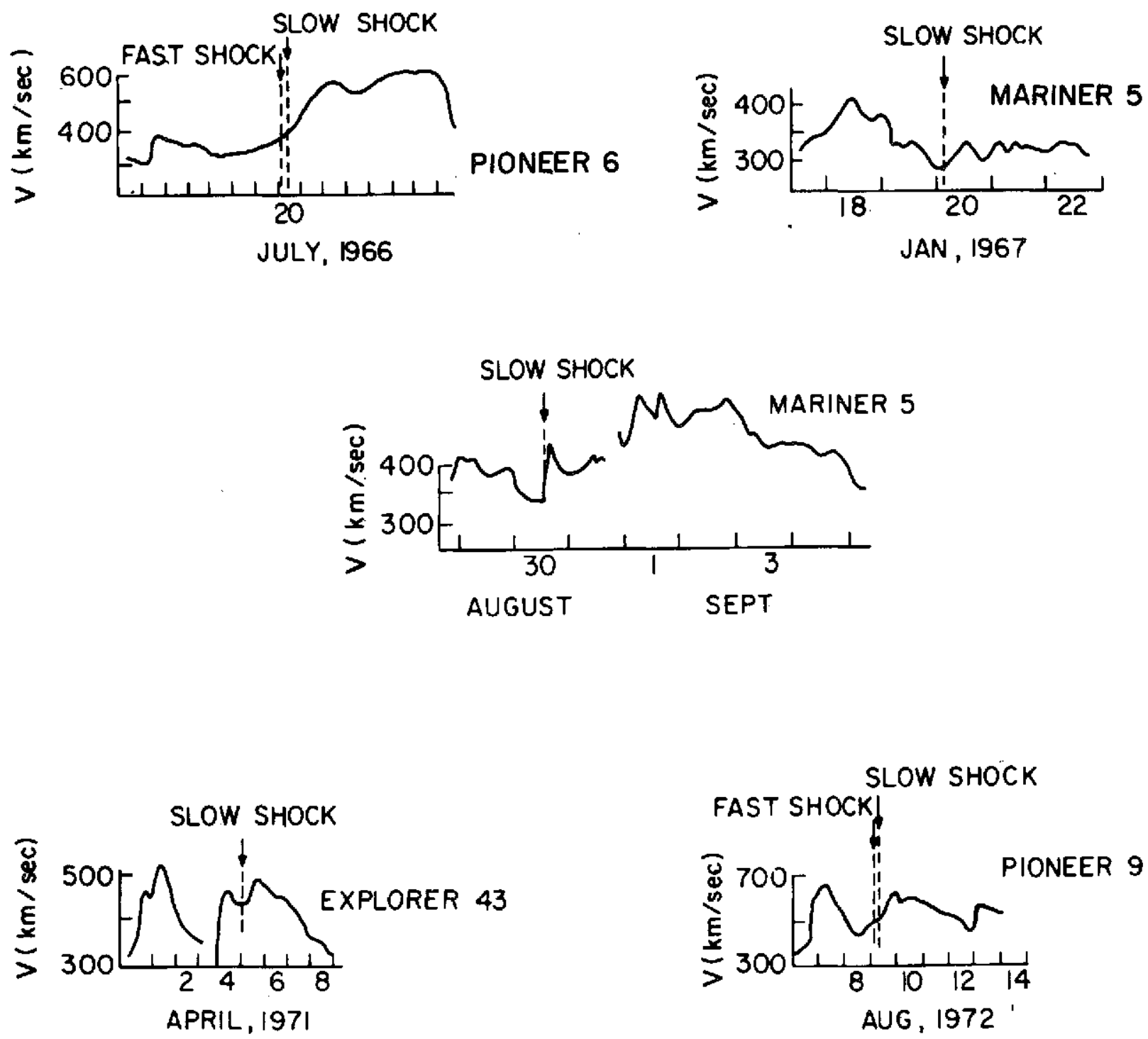